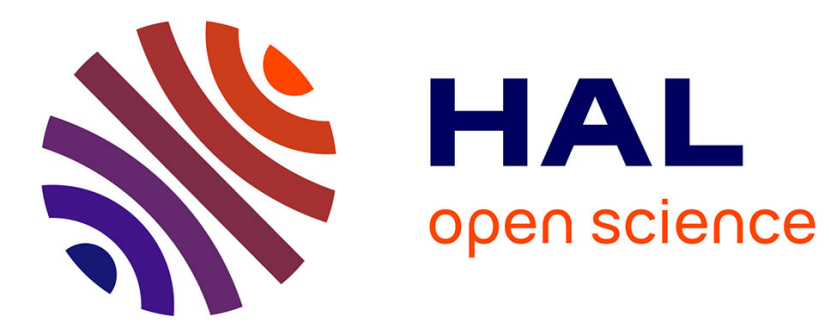

\title{
Heuristics and lower bounds for minimizing fuel consumption in hybrid-electrical vehicles
}

Sandra Ulrich Ngueveu, Stéphane Caux, Frédéric Messine, Mouloud Guemri

\section{To cite this version:}

Sandra Ulrich Ngueveu, Stéphane Caux, Frédéric Messine, Mouloud Guemri. Heuristics and lower bounds for minimizing fuel consumption in hybrid-electrical vehicles. 4OR: A Quarterly Journal of Operations Research, 2017, 15 (4), pp.407-430. 10.1007/s10288-017-0343-5 . hal-01671023

\section{HAL Id: hal-01671023 \\ https://hal.science/hal-01671023}

Submitted on 21 Dec 2017

HAL is a multi-disciplinary open access archive for the deposit and dissemination of scientific research documents, whether they are published or not. The documents may come from teaching and research institutions in France or abroad, or from public or private research centers.
L'archive ouverte pluridisciplinaire HAL, est destinée au dépôt et à la diffusion de documents scientifiques de niveau recherche, publiés ou non, émanant des établissements d'enseignement et de recherche français ou étrangers, des laboratoires publics ou privés. 


\title{
Heuristics and lower bounds for minimizing fuel consumption in hybrid-electrical vehicles
}

\author{
Sandra Ulrich Ngueveu \\ LAAS-CNRS, Université de Toulouse, CNRS, INP, F-31400 Toulouse, France \\ ngueveu@laas.fr \\ Stéphane CAUX, Frédéric MESSINE, Mouloud GUEMRI \\ LAPLACE, UMR CNRS 5213, INPT, UPS, 2 rue Camichel, 31071 Toulouse, France \\ 2017: Published in journal 4OR-Q J Oper Res - doi 10.1007/s10288-017-0343-5
}

\begin{abstract}
In hybrid electric vehicles, the electrical powertrain system has multiple energy sources that it can gather power from to satisfy the propulsion power requested by the vehicle at each instant. This paper focusses on the minimization of the fuel consumption of such a vehicle, taking advantage of the different energy sources. Based on global optimization approaches, the proposed heuristics find solutions that best split the power requested between the multielectrical sources available. A lower bounding procedure is introduced to validate the quality of the solutions. Computational results show a significant improvement over previous results from the literature in both the computing time and the quality of the solutions.

Keywords: OR in energy, Hybrid-Electric Vehicles, Heuristics, Lower Bound, Global Optimization, Optimal control
\end{abstract}

\section{Introduction}

The growing interest in environment-friendly hybrid vehicles during the design of new cars, has opened several research fields such as energy management for power distribution. A hybrid vehicle operates with at least two energy sources: (i) an internal combustion engine, a fuel cell with a hydrogen tank or a solar panel; mostly a non-reversible source that can only furnish a limited quantity of power at each instant-time, combined with (ii) a reversible source such as a battery or a super-capacitor, able to store the energy generated during the braking phases and able to give it back later. The operation of such vehicles is restricted by several constraints depending on the chosen energy sources: fuel cells have a slow dynamic due to the air compressor as well as the internal flow and temperature control inside their stack; reversible sources have a higher cost, lower lifespan, and limited energy capacity which hinder their proliferation in vehicles. Nowadays, the storage element plays the role of a secondary energy source that supports the primary source but it represents an intermediate step towards full electrical vehicles ([Burke, 2007, Chan, 2007]). 
Several operational research approaches have been applied on power control and energy management problems ([Bosman et al., 2012, Jacomino and Le, 2012, Vis, 2006]) for hybrid electric vehicles ([Bernard et al., 2010, Hofman et al., 2007, Shen et al., 2011]). The goal is to minimize the energy consumption. If the power demand, at each instant-time of the mission, is known a priori, the best offline strategy is searched for. Otherwise, an online strategy has to be designed to allow real time optimization ([Gao et al., 2009, He et al., 2012]). This paper focusses on the first case, although the best offline algorithms, if fast enough, may be applicable in an online context, if necessary with short term rolling time windows, as well as past and future data based on predictions ([Ngo et al., 2010, Pérez and Pilotta, 2009]).

The remainder of the paper is organized as follows: Section 2 specifies the problem studied. In Section 3 an overview of classical solution methods from the literature is presented. Section 4 highlights the main weaknesses identified in the best known method from the literature. The proposed heuristics are described and detailed in Section 5. In Section 6 and 7, the lower bounding procedure is introduced and the results obtained are analyzed, before the Conclusion.

\section{Problem description}

The problem consists in finding at each instant the optimal power split between multiple energy sources to satisfy the power demand of a driver on a predefined road section. The objective is to minimize the total fuel consumption of the vehicle during the mission, taking into account the characteristics and the limitations of each energy source.

The hybrid electric system considered in this paper has two energy sources, as illustrated on Figure 1: a Fuel Cell stack (FC) and a Storage Element (SE) which is a pack of supercapacitors. It derives from a hybrid full electric vehicle classified as hybridization series. The FC produces electricity from hydrogen (fuel). The SE can produce energy or recover the energy generated during the braking phases, or from the FC, for a later reuse. The energy efficiency of each component is measured by the ratio between the amount of useful energy produced by the component and the total energy spent by the component. The amount of useful and usable energy is equal to the energy spent minus the energy losses. Figure 2 illustrates the efficiency curves of the FC and the loss curve of the SE which were obtained experimentally ([Hankache, 2008]), including power converter efficiencies and the local current/voltage control.

The goal is to minimize the overall cost of hydrogen consumption for the vehicle which follows a given profile of power demands. This is done by optimizing, for each instant-time of the mission, the distribution of the power on the two sources taking into account their constraints of availability, performance and the state of charge limitations.

\subsection{Data Description}

\subsubsection{Power demand}

Let $P_{\operatorname{dem}_{i}}$ be the power requested by the powertrain at instant $i$. It is positive when the system is in traction, and negative when the vehicle is braking. At the end of the mission, the power demand is zero and the vehicle is stopped. Figure 7(a) illustrates an example of power 


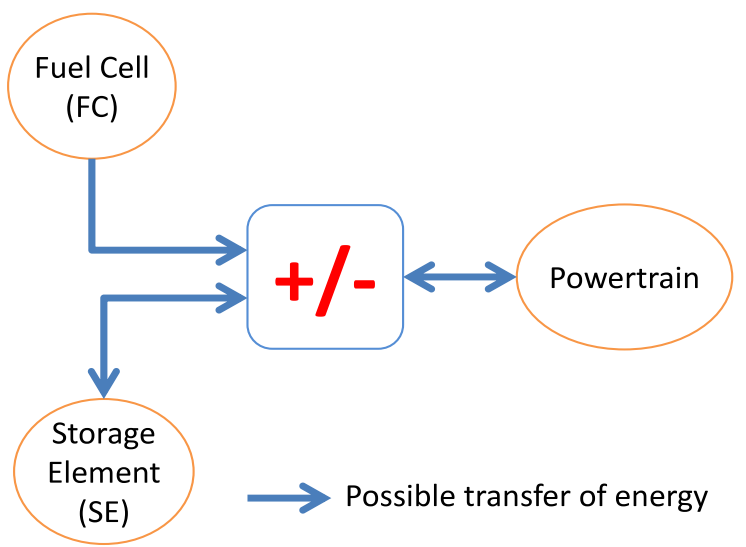

Figure 1: Series-hybrid architecture of the vehicle.

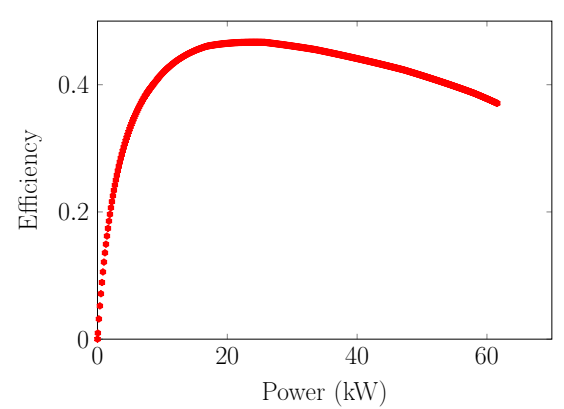

(a) Efficiency of the FC.

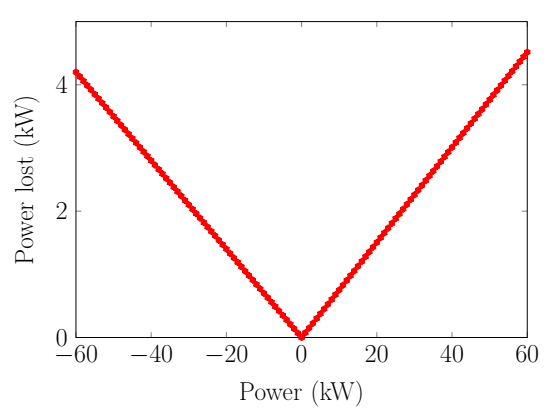

(b) Loss in the SE.

Figure 2: Efficiency curve of the FC and loss curve of the SE obtained experimentally 
profile which was provided by IFSTARR (French National Institute for Research on Transport and Transport Safety); it represents an electrical power demand in an urban environment.

\subsubsection{Power and energy bounds}

The FC is non reversible and can produce power up to $P_{\mathrm{FC}_{\max }}$ at each instant. Conversely, the SE can retrieve power up to $P_{\mathrm{SE}_{\min }}$ and can produce power up to $P_{\mathrm{SE}_{\max }}$. In addition, the $\mathrm{SE}$ can only work if the quantity of energy it stores is between $E_{\mathrm{SE}_{\min }}$ and $E_{\mathrm{SE}_{\max }}$, typically $25 \%$ and $100 \%$ of its energy capacity [4]. The amount of energy stored in the SE is also called state of charge (SOC). The initial and final state of charge of the SE, if it is given, will be referred to as $E_{\mathrm{SE}_{\text {init }}}$ and $E_{\mathrm{SE}_{\text {final }}}$.

\subsubsection{Efficiency functions of the energy sources}

Let $x_{i}$ and $y_{i}$ be the continuous variables equal to the power produced respectively by the $\mathrm{FC}$ and by the SE at the instant-time $i$. Therefore, the hydrogen consumption cost at any instant-time $i$ is $f_{\mathrm{FC}}\left(x_{i}\right)$ where $f_{\mathrm{FC}}$ is a known function that takes into account the energy loss that happens during any energy transfer. Conversely, the instantaneous energy consumption of the storage element at instant $i$ is $f_{\mathrm{SE}}\left(y_{i}\right)$. Note that in the latter case, $f_{\mathrm{SE}}$ is considered to have a negligible or no dependence to the state of charge of the SE (that is usually the case for super-capacitors, but not for batteries).

Functions $f_{\mathrm{FC}}$ and $f_{\mathrm{SE}}$ are derived from the experimental efficiency data and the energy loss data illustrated on Figure 2. These functions include the power electronics as well as the ancillaries losses and efficiencies that occur within the FC stack and the super-capacitors pack.

\subsection{Mathematical model}

As proposed by [Pérez et al., 2006], the related optimal control problem can be formulated as follows:

$$
\begin{aligned}
& \min _{u} \int_{0}^{T} f_{\mathrm{FC}}(x(s)) d s \\
& \text { subject to } \quad \dot{y}=-f_{\mathrm{SE}}\left(P_{\mathrm{dem}}-x\right) \\
& y_{0}=E_{\mathrm{SE}_{\text {init }}} \\
& y_{T}=E_{\mathrm{SE}_{\text {final }}} \\
& 0 \leq x \leq P_{\mathrm{FC}_{\max }} \\
& P_{\mathrm{dem}}-P_{\mathrm{SE}_{\max }} \leq x \leq P_{\mathrm{dem}}-P_{\mathrm{SE}_{\text {min }}} \\
& E_{\mathrm{SE}_{\min }} \leq y \leq E_{\mathrm{SE}_{\max }}
\end{aligned}
$$

where the control variable $x_{t}$ represents the power produced by the $\mathrm{FC}$, the state variable

$y_{t}$ represents the state of charge of the SE. The objective-function (1) minimizes the total fuel (hydrogen) consumption all over the mission duration T. This optimal control problem has constraints (5)-(6) on the control action and bounds (7) on the state variable, therefore it is difficult to apply the Pontryagin maximum principle. 
Note that this problem is naturally time-discretized because $P_{\mathrm{dem}}, f_{\mathrm{FC}}$ and $f_{\mathrm{SE}}$ data are obtained experimentally and thus they are sampled. It is therefore possible, instead of interpolating the data points to obtain a continuous formulation of the problem, to directly solve its time-discretized version; where $P_{\mathrm{dem}_{i}}$ is the power required at instant $i$; with $i$ an integer varying from 1 to $n$; where $n$ is the number of data points obtained from an experimental sampling of a real driving mission. There is no loss of precision as long as all data points are used, especially since the converters need time (in relation to the internal control responsiveness) to establish current and to provide the energy requested from $t_{i}$ to $t_{i+1}$. For example, current and voltage are controlled with a time constant of about 10ms, and the power sampling used in power splitting is 1s. Therefore, under this time separation the current and voltage can be considered instantaneous and able to follow power variations every $1 \mathrm{~s}$.

\section{Literature review}

In order to solve the problem of minimizing the fuel consumption of a hybrid-electric vehicle following a given mission profile, some solution approaches focus on defining the behavior of the SE in terms of power delivered or SOC variation, that leads to a lowering of the cost of hydrogen consumed, and then use these SOC values to deduce the FC powers ([Hankache, 2008]). Other approaches try to directly compute the powers delivered by the FC and then use the values obtained to deduce SOC ([Pérez and Pilotta, 2009]). The remainder of this section presents an overview of the latests solution methods from the literature that have been applied on offline energy management problems for hybrid electric power systems in the general case.

It is important to note that despite the intensification of the researches on this field, Dynamic Programming (DP) is still considered as the reference method when no assumption of convexity or concavity can be made on some of the efficiency functions. This happens because it provided the best known results in the literature so far in terms of quality of the solutions found, notwithstanding the significant computational time required. Numerous alternative approaches have been proposed, often much faster than the DP, but to the best of our knowledge, none of them found solutions of better quality than the DP, when none of the efficiency functions could be assumed convex or concave. [Gaoua et al., 2013] for example proposed a reformulation for the special case where the efficiency function of the storage element is convex.

\subsection{Dynamic programming}

Dynamic Programming (DP) is a well known method which is used to solve a variety of optimization problems. For a detailed description see for example [Bertsekas, 2011]. In the energy management literature, the DP provided the best results in terms of quality of the solutions found, sometimes after an additional discretization of the data ([Brahma et al., 2000, Pérez and Pilotta, 2009, Shen et al., 2011, Yu et al., 2009]).

The DP is applied on a graph where each node represents a feasible pair (time step, energy level) or (time step, power level), and arcs connect nodes of consecutive time steps if it is possible for the vehicle to move from one energy level or power level to another in a single 
time step. A cost function $\operatorname{Cost}\left(k, j, j^{\prime}\right)$ is assigned to each arc and it corresponds to the fuel consumption spent by the vehicle to move from the energy level $j$ to the energy level $j^{\prime}$ during the time step $k$. The DP computes a minimal cost path between the initial node (time step , $^{\circ}$ initial energy level) and the final node (time $\operatorname{step}_{n}$, final energy level) on the resulting graph.

Let us recall that for the problem we address, any given mission profile specifies a power demand already discretized in function of time ("horizontally") because the data is provided after experimentations on vehicle prototypes. In addition, the converters need time to establish steady current and to provide the energy requested.

[Hankache, 2008] proposed a DP algorithm which required an additional discretization of SOC ("vertically") to generate the nodes of the graph on which the DP algorithm can be applied. In this way, it was also possible to enforce constraint (4) about the final SOC.

Several variants of dynamic programming such as the one in [Pérez et al., 2006] have been proposed to speed up the computations but not to improve the solution quality. In fact, the most part of these works propose to obtain slightly worse or equivalent solutions but with a much shorter computational time. Thus, to facilitate comparisons with methods unable to impose a predefined final SOC $\left(=E_{\mathrm{SE}_{T}}\right)$, penalties proportional to $\left(E_{\mathrm{SE}_{T}}-y_{T}\right)$ can be added to the fuel consumption cost before it is compared to the DP solution cost.

Note that the DP provides the optimal solution of the discretized problem it solves, but the quality of this solution with regards to the original problem, highly depends on the discretization and additional assumptions made a priori. This issue will be addressed in Section 4 .

\subsection{Equivalent Consumption Minimization Strategy}

This is a control strategy based on the minimization of the equivalent consumption. Briefly, a cost of solicitation of the SE is defined by equaling the SE to a secondary fuel tank. In this case, any discharge of the SE becomes equivalent to some energy consumption (positive fuel flow) and any recharging becomes equivalent to some energy refill (negative fuel flow). Therefore, the resulting strategy is to minimize the total equivalent consumption ([Paganelli et al., 2002]). It means to replace the objective-function with equation (8):

$$
\min \int_{0}^{T} f_{\mathrm{FC}}(x(s))+\alpha f_{\mathrm{SE}}\left(P_{\mathrm{dem}}(s)-x(s)\right) d s
$$

where $\alpha$ is a coefficient of equivalence.

The equivalent consumption minimization strategy (ECMS) uses the rules of the optimal control to solve the resulting problem: the Hamiltonian function is applied to find a minimum ([Gao et al., 2009, Pisu and Rizzoni, 2007, Shen et al., 2011]), although tuning and using it under constraints is not straightforward (as expressed in Subsection 3.3). The solution costs obtained in the literature are not better than the DP's, but the algorithm is applicable in an online varying context, contrary to the DP.

\subsection{Shooting techniques}

As shown in Subsection 2.2, the problem can be formulated as an optimal control problem with bounds and constraints on the control and on SOC variables. Different publications were 
based on the application of the Pontryagin maximum principle, for example to solve equation (8) but none provided a significant improvement compared to the DP ([Hankache, 2008, Kim et al., 2011, Shen et al., 2011]). Although, the solutions obtained could sometimes be easier to implement in an online or real-time context. In fact, [Kim et al., 2011] found an improvement of $0.07 \%$ but which was attributed to truncation errors.

[Pérez and García, 2010] specified the equations resulting from the application of the optimal conditions of the Pontryagin maximum principle and then emphasized on the difficulties that hindered to the solution of these equations. As an alternative, [Pérez and García, 2010] proposed a direct shooting technique so-called Direct Transcription (DT) which consists in discretizing the problem over the time horizon and then solving the resulting non-linear formulation with a projected augmented Lagrangian algorithm. No comparison to the literature or previous publications was provided and the authors mentioned the high sensitivity of the resulting code to parameters settings. Then, they proposed a linear constrained approximation of the problem whose solution could be used as a starting point to help to reduce the computational times.

\subsection{Rule-based algorithms}

Rule-based algorithms have been proposed for the energy management in hybrid vehicles ([Hofman et al., 2007, Jalil et al., 1997, Shen et al., 2011]), among which the method so-called "thermostat". Its principle is to turn the combustion engine on or off based on the SOC status as follows: (i) if SOC reaches a predefined lower value then the engine is turned on; (ii) if a predefined higher value is reached, the engine is turned off, and it stays off until the predefined SOC lower value is reached and the cycle repeats itself. Fuzzy logic ([Zadeh, 1965]) has also been used, but mainly for online management with offline tuning optimisation, taking advantage of its inherent ability to adapt the rules in function of the evolution of the profile of power demand ([Neffati et al., 2012]).

However, none of these rule-based algorithms produced solutions of lower cost than the DP's solutions. The rule-based algorithms of [Hofman et al., 2007] found solutions about $1 \%$ worse than the DP ones but these methods were applicable in an online context.

\section{Main weaknesses identified in dynamic programming}

Despite the various approaches and methods from the literature, the DP is always presented as the one which provides the best results in term of quality of the obtained solutions, although sometimes requiring intensive computational efforts. Therefore, there is a widespread perception that the DP-based algorithms from the literature provide the optimal solution of such problems as it is stated in [Pisu and Rizzoni, 2007]. However, several other flaws can be identified in these algorithms. These flaws originate from the implicit hypothesis or assumptions made in the literature when the DP is applied to solve the fuel consumption minimization problem. 


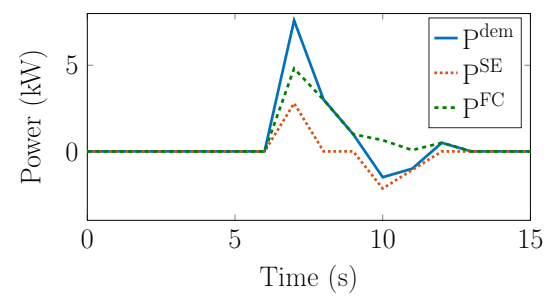

(a) All braking energy is recovered: cost (b) of the mission $=56.46 \mathrm{kWs}$

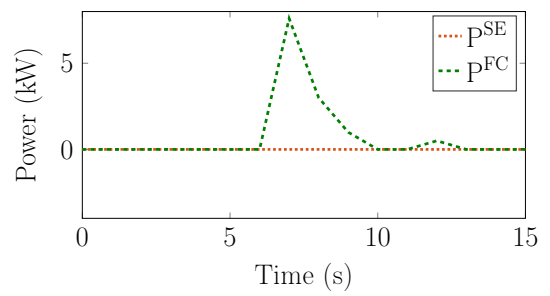

(b) No braking energy is recovered: cost of the mission $=46.96 \mathrm{kWs}$

Figure 3: Contrary example where all braking energy should not be recovered.

\subsection{Recovery of energy braking}

It is obvious that recovering energy from the braking phases for a later reuse can help minimizing the fuel consumption cost of a mission. However, contrary to the common assumption, imposing that all braking energy has to be recovered may lead to worse solutions, especially if it is applied with the DP with constraints on the final SOC.

Figure 3 illustrates an example on a 15 seconds profile, where $P_{\mathrm{SE}}$ and $P_{\mathrm{FC}}$ are the power provided by respectively the $\mathrm{SE}$ and the FC. In the first case, when retrieving braking energy, the cost of the mission is equal to $56.46 \mathrm{kWs}$. In the second case, no braking energy is recovered and the cost is reduced to $46.96 \mathrm{kWs}$. Unrecovered braking energy can be simply dissipated as heat.

Recall that the DP requires a full discretization of the energy level. Imposing that all breaking energy must be recovered means enforcing $P_{\mathrm{dem}}=P_{\mathrm{FC}}+P_{\mathrm{SE}}$. As a consequence the FC has to provide energy because all values of $P_{\text {dem }}$ cannot always be exact multiples of the $P_{\mathrm{SE}}$ step, and therefore there is no feasible solution where $P_{\mathrm{dem}}=0+P_{\mathrm{SE}}$ on all the braking phases.

Another counter-example can be generated by considering a vehicle descending a downhill path. In this case, the amount of braking energy is greater than the amount of traction, therefore there is no feasible solution that allows to recover all the braking energy. As a consequence, the widely used equality constraint $P_{\mathrm{dem}_{i}}=x_{i}+y_{i}$ should be replaced with the inequality $P_{\mathrm{dem}_{i}} \leq x_{i}+y_{i}$. This means that the power demand must always be satisfied, but during the braking phases, the recovery of all or of a part of the energy generated is authorized but not mandatory.

\subsection{Final state of charge of the SE}

To facilitate comparisons between different algorithms and to ensure that the vehicle can perform repetitive missions, the papers from the literature often impose the final state of charge of the $\mathrm{SE}$ to be equal to the initial state of charge $\left(E_{\mathrm{SE}_{0}}=E_{\mathrm{SE}_{T}}\right)$. This is contrary to the charge-depleting mode that disregards the energy in the storage element at the end, usually for plug-in vehicles which can reload the SE on the grid at the end of their mission.

However, for some instances, such "energy-balancing" constraint means that the vehicle had to consume excess energy towards the end of the profile in order to return the energy level to its initial state. This means that when the FC is used, it is at a very small power 

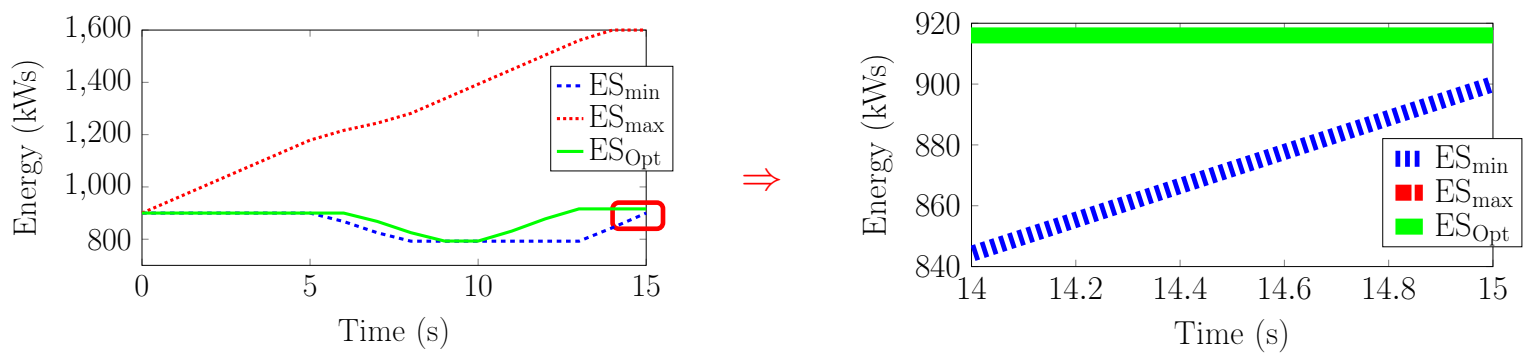

Figure 4: Excess energy at the end (final SOC greater than the starting SOC).

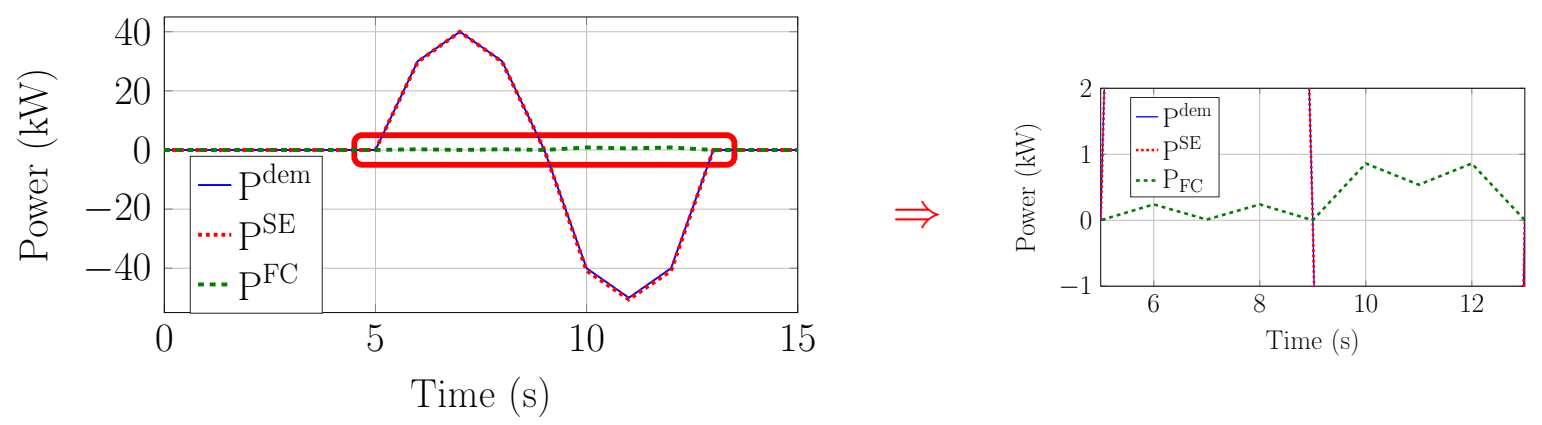

Figure 5: Penalties during the optimization

level, which corresponds to the poorest efficiency coefficients. This results into a higher total consumption.

The solution proposed in this work to ensure repetitive missions but avoid the drawback described in the previous paragraph, is to forbid the vehicle to return the SE at the end of its mission with less energy than the quantity it started with, but to allow the final energy level to be higher than at its start (but with no special reward for the additional energy). In a DP algorithm, this corresponds to opening the validity domain and allowing the final state of the SE to vary in an interval instead of being reduced to a single point (see for example Figure 4). Note that this is not a charge-depleting mode, because the SE is not allowed to end with less energy than at start. The excess of energy may be dissipated in a resistance.

\subsection{Penalties due to the SE discretization}

Figure 5 illustrates an example where, although the braking energy is greater than the traction energy, which should lead to a cost of zero (no need to consume any fuel), the DP produces a solution which uses the FC and therefore has a cost strictly positive. Basically, since the power levels have been discretized, the system tends to respond too strongly to demands that are not exact multiples of the discretization step. This flaw, which would require excessively small discretization steps and thus a prohibitive computational time to be mitigated (see for example Table 1), has been handled when designing the new heuristics proposed.

To summarize, although the DP produces the best solutions in the literature when the efficiency functions are neither convex or concave, there is an increasing need for new and more efficient approaches to find better solutions, first in terms of quality of the solutions and 


\begin{tabular}{|c|c|c|c|c|c|}
\hline$\delta_{E}(\mathrm{~kW})$ & 5 & 3 & 2 & 1 & 0.5 \\
\hline Consumption cost $(\mathrm{kWs})$ & 10142.8 & 10133.8 & 10131.6 & 10130.6 & 10130.2 \\
\hline Computing time $(\mathrm{min})$ & 3.4 & 9.6 & 22.1 & 87.95 & 350.9 \\
\hline
\end{tabular}

Table 1: Impact of the SE discretization step on solution cost and computing time

additionally, if it is possible, in terms of computing time, although it may not be the main concern in an offline optimization context.

\section{New Heuristic Methods}

\subsection{Heuristic 1: Filtering bands}

The principle of this heuristic is inspired from electrical filters: filtering is applied on the power demand to determine when the power should be supplied by the FC (low power variation) and when it should be supplied by the SE (able to provide/recover quick power variations). It requires two parameters $B_{l}$ and $B_{u}$ named respectively lower and upper bands:

- $B_{l}$ is chosen between 0 and the $P_{F C \max }$

- $B_{u}$ must be strictly higher than $B_{l}$ and less than $P_{F C \max }$.

The core of this heuristic is summarized with Algorithm 1. Notice that the SE is never charged directly by the FC and the validity domain must be open. In Figure 6 , it is shown how the bands are selected with regards to the efficiency function.

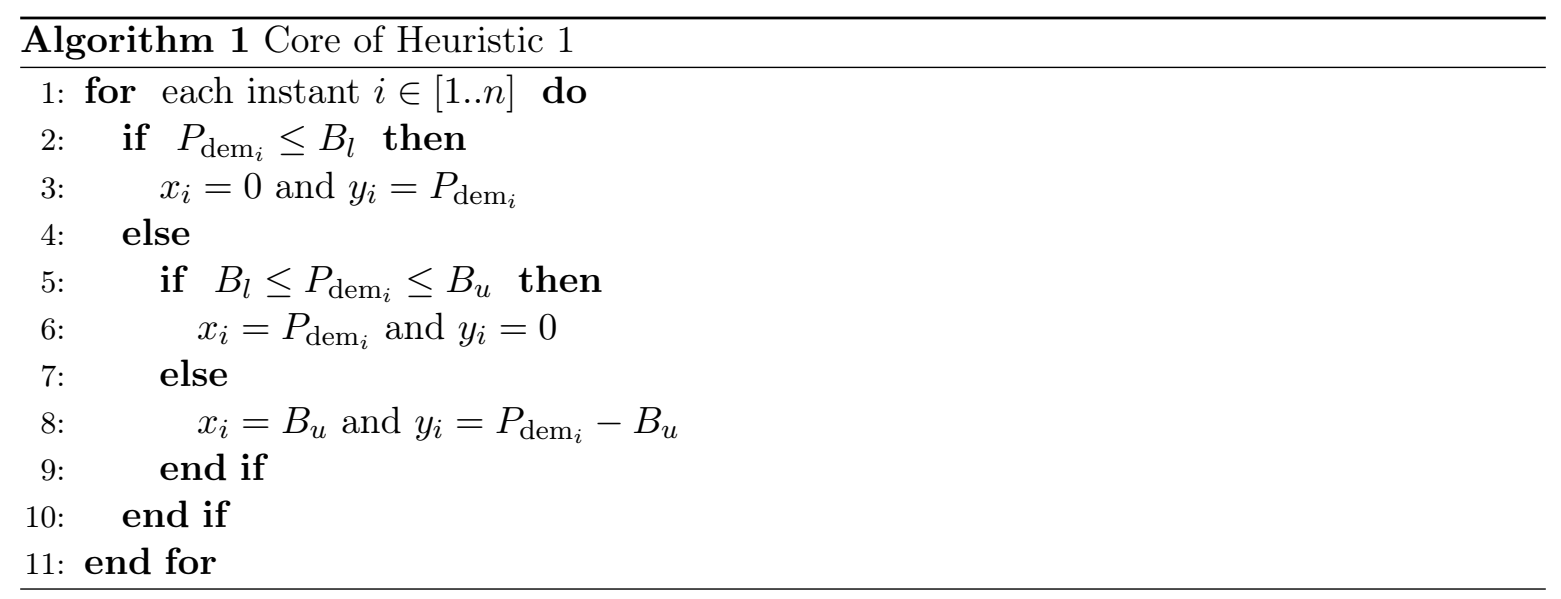




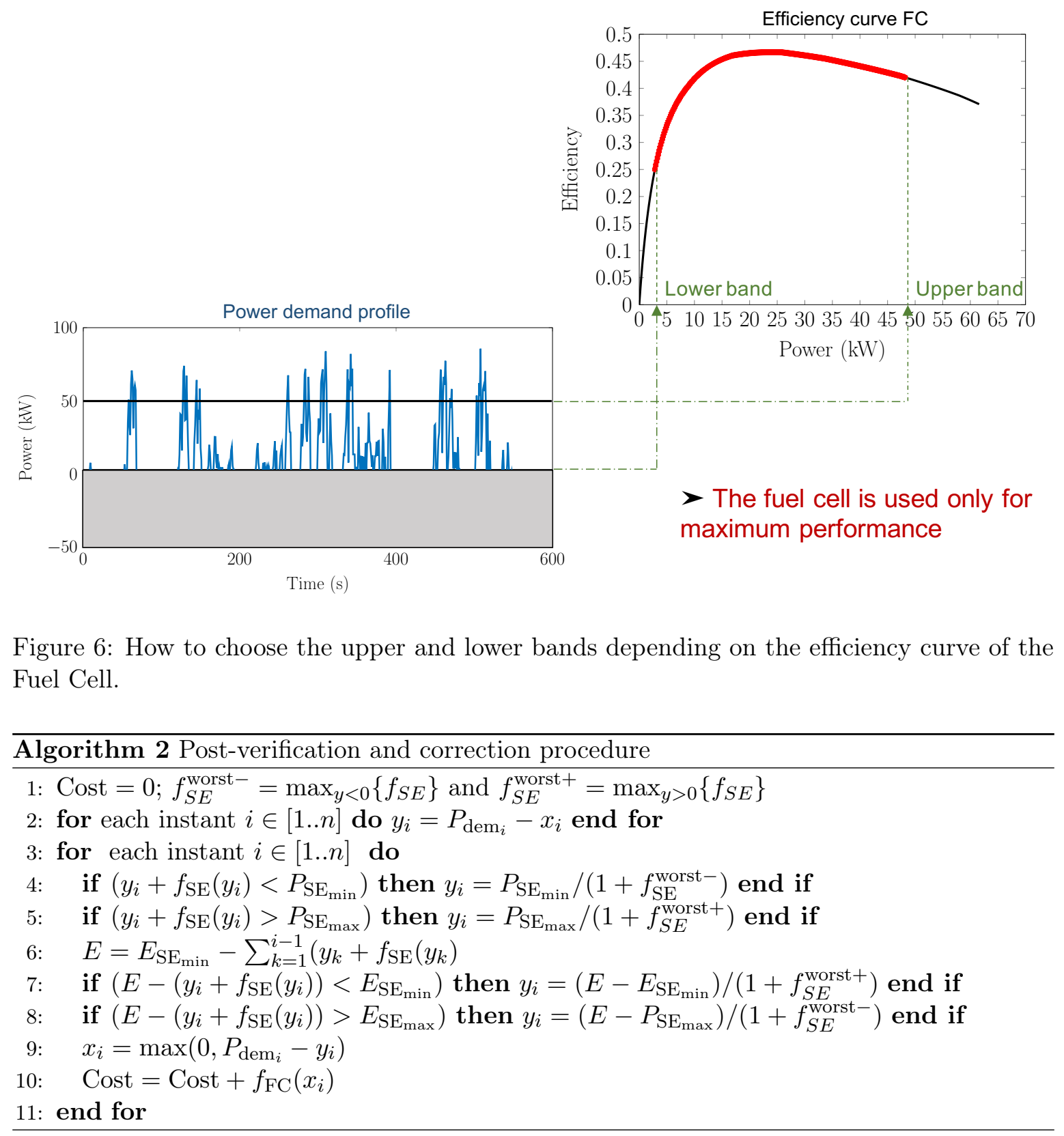

This approach has several advantages:

(i) Because of its short computational time (few seconds for a 600 samples mission), it is possible to run several tests and fine-tune the parameter settings.

(ii) A simple modification of the interval $\left[B_{l}, B_{u}\right]$ ensures that the desired final SOC is reached.

(iii) The best solutions obtained by such a strategy are reached after a few iterations, and then submitted to Algorithm 2 for either a post-verification that the variation of SOC 
remains in the domain of validity (7) or a correction of the solution which ensures that it becomes feasible.

The difference between this heuristic and the well-known method "Thermostat" ([Jalil et al., 1997]) is that the latter filtered the power demand by looking at the SE whereas this heuristic focusses on the FC to bypass the issue described in Subsection 4.3. To summarize, the SE turns on when the power demand is outside the chosen bands and turns off otherwise, whereas the FC is turned off for low power demand and does not exceed the chosen bands which usually corresponds to high FC efficiency coefficients, as shown in Figure 6. Note that here the SE is always charged when $P_{\text {dem }}<0$.

The choice of bands $B_{l}$ and $B_{u}$ can be left to an experienced engineer, and satisfactory results can be obtained for each instance in a few attempts. Nevertheless, in the computational evaluation, it is assumed that the user could not provide satisfactory band values as an input. In this case, an iterative procedure is applied where the values of $B_{l}$ and $B_{u}$ are initially set at $B_{l}^{\text {init }}$ and $B_{u}^{\text {init }}$ and Algorithm 1 is applied. Then the values of the bands are respectively increased and decreased until the application of Algorithm 1 does not result into a feasible solution in terms of final state of charge; i.e. until the final state of the storage element drops below the value of its initial state. The two initial values verify $B_{l}^{\text {init }} \leq B_{u}^{\text {init }}$, $f_{\mathrm{FC}}\left(B_{l}^{\text {init }}\right) \geq f_{\mathrm{FC}}^{\text {best }}, f_{\mathrm{FC}}\left(B_{u}^{\text {init }}\right) \geq f_{\mathrm{FC}}^{\text {best }}, f_{\mathrm{FC}}\left(B_{l}^{\text {init }}-\Delta\right) \leq f_{\mathrm{FC}}^{\text {best }}$ and $f_{\mathrm{FC}}\left(B_{u}^{\text {init }}+\Delta\right) \leq f_{\mathrm{FC}}^{\text {best }}$ where $f_{\mathrm{FC}}^{\text {best }}$ is a best known value of $f_{\mathrm{FC}}$ and $\Delta$ is a parameter defining a step size. The final solution of the resulting procedure is the best feasible (in terms of final state of charge) solution produced during the different iterations. Heuristic 1 returns the solution obtained after application of Algorithm 2 on that solution.

\subsection{Heuristic 2: DP with discretization of $P_{\mathrm{dem}_{i}}$ before the SE}

In most papers, as in [Hankache, 2008], the application of the DP is done after discretizing the time horizon and SOC in order to generate the graph. It has several drawbacks, as described in Section 4. The problem data are already discretized in terms of time as explained at the end of Subsection 2.2. One solution is to discretize the FC instead of the SOC variation, as in [Pérez and Pilotta, 2009]. The idea is to let the SE absorb any noises or errors in cases where the demand is not exactly proportional to the discretization step, instead of the FC, in order to limit the resulting unnecessary fuel consumption. However such approach results into the computation of a resource constrained shortest path (where the SOC value is the resource), instead of a shortest path. This translates into worse computing times.

As an alternative, Heuristic 2 keeps the SOC variation discretization $(\Delta \mathrm{SOC})$ with its benefits in terms on computing times, but uses equation (9) to preprocess the demand profile and to generate a new profile that takes into account the SOC discretization. Any solution computed with the new profile is feasible for the original profile since the excess of energy can be simply dissipated as heat.

$$
\bar{P}_{\operatorname{dem}_{i}}=\left\lfloor\left(P_{\operatorname{dem}_{i}}+\Delta \mathrm{SOC}\right) / \Delta \mathrm{SOC}\right\rfloor \times \Delta \mathrm{SOC}
$$

Note that in this case, the solution algorithm and recursive formula remain the same as the ones from the literature; only the input data has been pre-processed with equation (9) before the application of the algorithm. 


\subsection{Heuristic 3: Relaxing braking energy recovery}

For the DP from [Hankache, 2008], as it is often the case in the literature, the SE is required to collect all the braking energy generated. As explained in Subsection 4.1, this may lead to additional fuel consumption and thus an increased cost of the mission. The goal of Heuristic 3 is to collect only the amount of energy that would not deteriorate the quality of the final solution. As a consequence, the following constraint is relaxed for all instant times $i \in I$ and $\varepsilon_{i} \geq 0$ :

$$
\begin{aligned}
& \text { Classical equation : } x_{i}+y_{i}=P_{\mathrm{dem}_{i}} \\
& \text { New equation : } x_{i}+y_{i}-\varepsilon_{i} \stackrel{\Downarrow}{=} P_{\mathrm{dem}_{i}} .
\end{aligned}
$$

The idea is to get less energy from braking and to provide more than the requested energy at some instants if it leads to a better efficiency coefficient (yielding a better solution). The excess of electrical energy $\varepsilon_{i} \geq 0$ can be dissipated as heat, in a resistance inserted in the braking system or in mechanical brakes present for security reasons. The resulting algorithm consists in applying the DP on a graph where the arc costs have been modified in accordance with the new equation. It can also be done by adding new edges to the graph.

\subsection{Heuristic 4: Local search from a predefined starting point using a gradient-based algorithm}

The problem can be reformulated as a non-linear problem taking into account its natural time-discretization as follows:

$$
\min \sum_{i=0}^{n} f_{\mathrm{FC}}\left(x_{i}\right)=\sum_{i=0}^{n} \frac{x_{i}}{\rho\left(x_{i}\right)}
$$

subject to

$$
\begin{array}{rr}
x_{i}+y_{i} \geq P_{\mathrm{dem}_{i}}, & \forall i \in[1 \ldots n] \\
\sum_{i=1}^{n}\left(y_{i}+\widetilde{\rho}\left(y_{i}\right)\right) \leq 0 & \\
\sum_{k=1}^{i}\left(y_{k}+\widetilde{\rho}\left(y_{k}\right)\right) \leq E_{\mathrm{SE}_{0}}-E_{\mathrm{SE}_{\min }} & \forall i \in[1 \ldots n] \\
\sum_{k=1}^{i}\left(y_{k}+\widetilde{\rho}\left(y_{k}\right)\right) \geq E_{\mathrm{SE}_{0}}-E_{\mathrm{SE}_{\max }} & \forall i \in[1 \ldots n] \\
0 \leq x_{i} \leq P_{\mathrm{FC}_{\max }}, & \forall i \in[1 \ldots n] \\
P_{\mathrm{SE}_{\min }} \leq y_{i} \leq P_{\mathrm{SE}_{\max }}, & \forall i \in[1 \ldots n]
\end{array}
$$

where $\rho\left(x_{i}\right)$ is the efficiency coefficient of the FC when producing $x_{i}$, whereas $\widetilde{\rho}\left(y_{i}\right)$ denotes the energy losses from the SE when producing or collecting a power of $y_{i}$. The curves $\rho$ and $\widetilde{\rho}$, obtained experimentally, are the ones given on Figure 2. 
Note that it is possible to force the recuperation of all the braking energy by replacing constraint (12) with classical constraint (18), although it may lead to worse solutions as discussed in Section 4. Likewise, it is possible to replace constraint (13) with (19) to force the equality between the initial SOC and the final SOC.

$$
\begin{aligned}
& x_{i}+y_{i}=P_{\operatorname{dem}_{i}}, \quad \forall i \in[1, \ldots n] \\
& \sum_{i=1}^{n}\left(y_{i}+\widetilde{\rho}\left(y_{i}\right)\right)=0 .
\end{aligned}
$$

Heuristic 4 (H4) consists in applying gradient-based optimization on the reformulation. Active-set, sqp or interior-point are examples of gradient optimization methods taking into account non-linear constraints. They are therefore used as a local search technique applicable from a predefined starting point. The idea is similar to [Pérez and García, 2010], however, the main challenge of such an approach is that, as a local search approach, its efficiency strongly depends on the chosen starting point. A starting point of $\mathrm{H} 4$ is in fact a real vector of dimension $2 n$ where $n$ is the number of considered instant-times. It therefore consists in real values for $x_{i}$ and $y_{i}$ with $i=1, \ldots, n$. In H4 ${ }^{\text {pdem }}$ (see below) for example, this vector is equal to the power demand at each instant-time. The use of random starting points leads to poor results and an increased computational time. Finding the best starting point is a key. Two variants of $\mathrm{H} 4$ were considered, depending on the chosen starting point:

- H4 $4^{\mathrm{pdem}}:$ uses a predefined starting point corresponding exactly to the power demand $P_{\mathrm{dem}_{i}}$, positive values assigned to $x_{i}$ and negative values assigned to $y_{i}$

- $\mathrm{H} 4^{\mathrm{H} 1}$ : uses a predefined starting point corresponding exactly to the solution obtained by Heuristic 1 which has short computational time, especially once the bounds have been set.

\section{Lower bounding procedure}

An upper bound (UB) of the consumption can be obtained by using only the fuel cell to satisfy all the requested positive powers while disregarding the negative power demands. All solution costs can be compared to this upper bound as it is done in the literature. Such upper bound corresponds to the energy consumption that the vehicle would require if it did not benefit from an additional reversible energy source that can store and can return energy.

However, to have more certainties on the quality of the solutions obtained for the hybridelectric vehicle, it would be better to evaluate their gap with the optimal solution. Since the optimal solution is yet unknown, a good lower bound is a satisfactory alternative.

For the problem studied in this paper, such a lower bound (LB) can be computed by assuming ideal conditions: that the FC efficiency remains at its maximum level. Assuming that $\rho\left(x_{i}\right)=\max _{x_{i}}\left\{\rho\left(x_{i}\right)\right\}=\alpha$ (constant), the objective-function (11) becomes the following linear function:

$$
\min \sum_{i=0}^{T} f_{\mathrm{LB}}\left(x_{i}\right)=\frac{x_{i}}{\alpha}
$$


The resulting problem has a linear objective-function, linear constraints and non-linear constraints. Because the SE energy loss function $\widetilde{\rho}$ from figure 2(b) is linear, the resulting problem can be easily solved with a linear solver. Note that if $\widetilde{\rho}$ is convex, then constraints (14) are convex and linear relaxations of constraints (13) and (15) can be obtained by taking into account the bounds (17) on $y_{i}$ ([Horst and Tuy, 2000]). The resulting problem is entirely convex and can be solved optimally using simply a gradient-based algorithm and thus a valid lower bound of the original problem is provided. Otherwise, a convex hull of the realizable domain should be constructed to ensure that the obtained solution is a lower bound of the original problem ([Horst and Tuy, 2000]).

\section{Computational analysis}

\subsection{Instances and data sets}

The computational evaluation of the offline algorithms developed was performed for a hybridelectric vehicle whose powertrain measurements are presented in Table 2 and the characteristics of power sources are illustrated in Figure 2. Six different mission profiles were used, illustrated on Figure 7 and derived from six different test drive cycles:

- INRETS: real-world profile provided by IFSTARR (French National Institute for Research on Transport and Transport Safety)(Figure 7(c)). It represents the power demand profile of electric vehicles in urban environments. The profile has a total duration of 561 s with a time step of 1 s which provides $n=561$ instants.

- ESKISEHIR: real-world profile provided by ALSTOM, corresponding to a Turkish tramway. The total duration is 1400 s with a time step of 1 s which provides $n=1400$ instants.

- URBAN: real-world profile provided by the LAPLACE (Laboratory on PLAsma and Energy Conversion) which represents a power demand profile in a urban environment. The total duration is 800 s with a time step of 1 s which provides $n=800$ instants.

- HIGHWAY: real-world profile provided by the LAPLACE which represents a power demand profile on a highway. The total duration is 750 s with a time step of $1 \mathrm{~s}$ which provides $n=750$ instants.

- NEDC: real-world profile derived from the New European Driving Cycle from the UNECE (United Nations Economic Commission for Europe). It represents the typical usage of a car in Europe. The total duration is 1200 s with a time step of 1 s which provides $n=1200$ instants.

- SYNTH: synthetized profile provided by [Pérez and García, 2010] which comes from an elementary velocity cycle. It resulted from measurements on a vehicle performing a 10 s constant acceleration until reaching a speed of $30 \mathrm{~km} / \mathrm{h}, 10 \mathrm{~s}$ at this speed, a $10 \mathrm{~s}$ constant deceleration and then no power demand until it was completely stopped. The total duration is 40 s with a time step of 1 s which provides $n=40$ instants. 


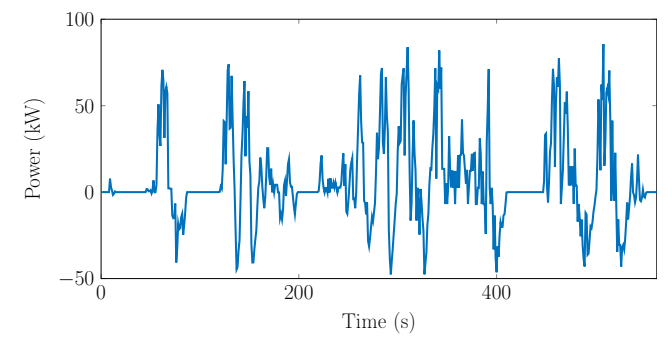

(a) INRETS profile.

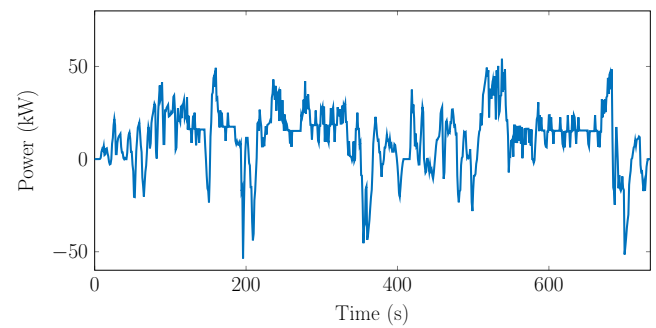

(c) HIGHWAY profile.

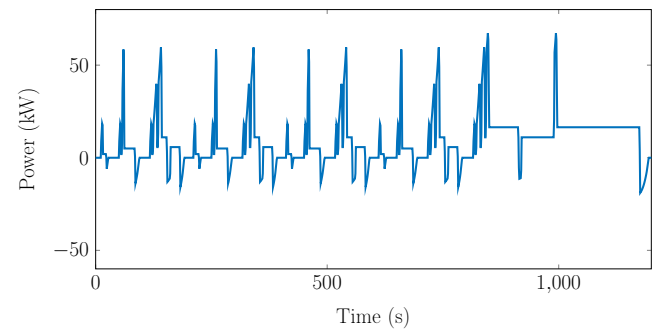

(e) NEDC profile.

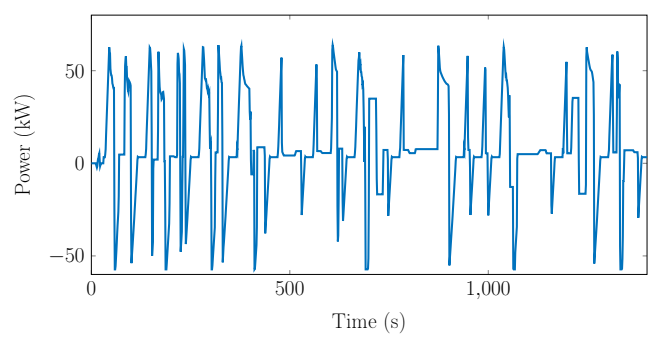

(b) ESKISEHIR profile

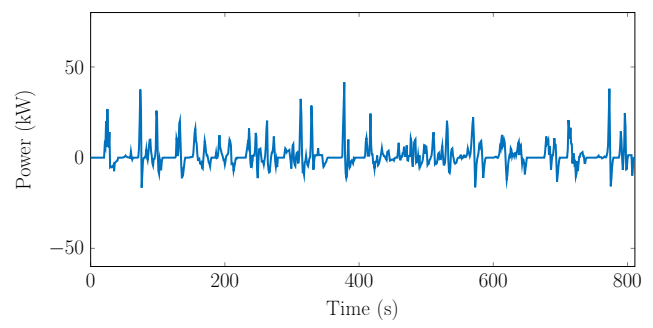

(d) URBAN profile.

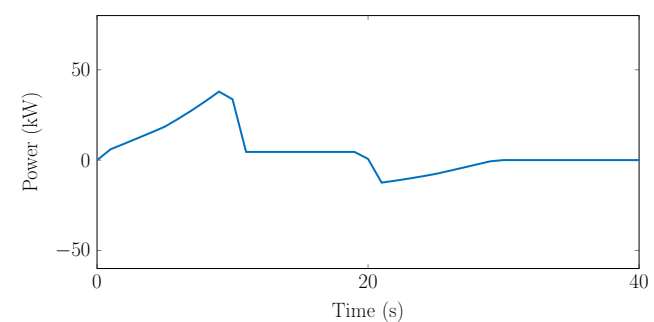

(f) SYNTH profile

Figure 7: Power demand profiles of the real-world instances (a)-(e) and the synthetized instance (f).

\begin{tabular}{|c|c|c|}
\hline Name & Description & Value \\
\hline$P_{\mathrm{SE}_{\min }}$ & min power extractible from the SE at any instant-time & $-60 \mathrm{~kW}$ \\
\hline$P_{\mathrm{SE}_{\max }}$ & max power extractible from the SE at any instant-time & $60 \mathrm{~kW}$ \\
\hline$E_{\mathrm{SE}_{\min }}$ & min energy level authorized in the SE at any instant-time & $400 \mathrm{kWs}$ \\
\hline$E_{\mathrm{SE}_{\max }}$ & max energy level authorized the SE at any instant-time & $1600 \mathrm{kWs}$ \\
\hline$P_{\mathrm{FC}_{\max }}$ & max power deliverable by the FC at any instant-time & $70 \mathrm{~kW}$ \\
\hline
\end{tabular}

Table 2: Characteristics of hybrid-electric vehicle used 


\subsection{Parameter settings and Table headings}

All heuristics have been programmed with MATLAB R2015b 64 bits ([Mathworks, 2013]) and run in the single computing thread mode on a desktop computer Intel(R) Xeon(R) $3.60 \mathrm{GHz}$ CPU E3-1271 v3 with 32 GB of RAM. Heuristic 4 and the lower bound use the non-linear solver 'fmincon' from the Optimization Toolbox. In both cases, the efficiency function can be represented in the form of a linear interpolation or a polynomial representation.

In Table 3, the results of the DP and the DT from the literature ([Hankache, 2008, Pérez and García, 2010]) compared to the proposed heuristics and lower bound on all mission profiles are summarized using the following headings:

- DP: Dynamic Programming from the literature, code implemented by [Hankache, 2008].

- DT: Direct Transcription solution method from [Pérez and García, 2010]. From the algorithm description, it can be infered that it is similar to initializing $\mathrm{H} 4$ with the linear constrained approximation of the problem.

- H1, H2, H3, H4 $4^{\text {pdem }}, \mathrm{H} 4^{\mathrm{H} 1}$ : The proposed heuristics, described in Section 5.

- UB: Upper Bound obtained assuming that only the FC is used. It corresponds to the fuel consumption of the vehicle if it was not a hybrid one, i.e., if there was no SE.

- LB: Lower Bound proposed, described in Section 6. It can be computed directly because $\widetilde{\rho}$ is linear on the instances.

- cost: Solution cost $=$ total hydrogen consumption in $\mathrm{kWs}$.

- time: Execution Time $=$ CPU time in seconds. Note that for H1, the running time reported is the one where the band values are to be determined with an iterative procedure. The typical running time for a predefined band (1 iteration) is less than five milliseconds.

- gaptoLB: Gap to the lower bound $=100 \times \frac{\text { cost }-\mathrm{LB}}{\mathrm{LB}}$.

In Table 4, the characteristics of the solutions found for the INRETS profile are detailed with the following additional headings:

- $\mathrm{HY}=$ Hydrogen storage: Total amount of hydrogen that has been inserted in the SE from the FC in the form of electricity, in kWs.

- $\mathrm{HL}=$ Hybridization Level (HL): Percentage of participation of the storage element in the traction power train (positive power required), computed with equation (21). It is used as a post-optimization classification criterion for hybrid vehicles, see among others ([Bolvashenkov et al., 2006, Buecherl et al., 2009, Lukic and Emadi, 2004]).

$$
\mathrm{HL}=\frac{\text { Total traction power of storage element }}{\text { Total traction power of all power sources }}
$$




\begin{tabular}{|c|c|c|c|c|c|c|c|c|}
\hline \multirow[t]{2}{*}{ Profile $(\mathrm{P})$} & \multirow[t]{2}{*}{ UB } & \multirow[t]{2}{*}{$\mathrm{LB}$} & \multicolumn{3}{|c|}{$\mathrm{DP}$} & \multicolumn{3}{|c|}{$\mathrm{DT}$} \\
\hline & & & cost & gap & time $(\mathrm{s})$ & cost & gap & time $(s)$ \\
\hline INRETS (I) & 15902.5 & 8647.0 & 10130.6 & $17.2 \%$ & 5516.5 & 11103.8 & $28.4 \%$ & 61.6 \\
\hline ESKISEHIR (E) & 47568.9 & 26529.0 & 31438.3 & $18.5 \%$ & 15240.5 & 30921.1 & $16.6 \%$ & 1461 \\
\hline URBAN (U) & 5815.1 & 2544.6 & 5986.3 & $135.2 \%$ & 5631.3 & 2855.2 & $12.2 \%$ & 480.8 \\
\hline HIGHWAY (H) & 23083.5 & 18395.1 & 20099.4 & $9.3 \%$ & 7793.2 & 19920.3 & $8.3 \%$ & 825.4 \\
\hline NEDC $(\mathrm{N})$ & 27903.4 & 22583.2 & 25127.8 & $11.3 \%$ & 13322.0 & 27032.3 & $19.7 \%$ & 323.8 \\
\hline SYNTH (S) & 621.9 & 432.1 & 554.1 & $28.2 \%$ & 35.5 & 630.8 & $46.0 \%$ & 0.2 \\
\hline & $\begin{array}{l}\text { n realisti } \\
\text { avg on al }\end{array}$ & $\begin{array}{l}\text { nstances } \\
\text { nstances }\end{array}$ & & $\begin{array}{l}38.3 \% \\
36.6 \% \\
\end{array}$ & $\begin{array}{l}9500.7 \\
7923.2 \\
\end{array}$ & & $\begin{array}{l}17.0 \% \\
21.9 \% \\
\end{array}$ & $\begin{array}{l}630.5 \\
525.5 \\
\end{array}$ \\
\hline
\end{tabular}

\begin{tabular}{|c|c|c|c|c|c|c|c|c|c|}
\hline \multirow[t]{2}{*}{ (P) } & \multicolumn{3}{|c|}{ H1 } & \multicolumn{3}{|c|}{$\mathrm{H} 2$} & \multicolumn{3}{|c|}{ H3 } \\
\hline & cost & gap & time $(\mathrm{s})$ & cost & gap & time $(\mathrm{s})$ & cost & gap & time $(\mathrm{s})$ \\
\hline (I) & 8840.9 & $2.2 \%$ & 0.1 & 9250.5 & $7.0 \%$ & 6029.1 & 9009.6 & $4.2 \%$ & 7933.0 \\
\hline (E) & 28318.8 & $6.8 \%$ & 0.6 & 28741.9 & $8.3 \%$ & 17750.5 & 28028.2 & $5.6 \%$ & 23297.8 \\
\hline (U) & 2685.7 & $5.5 \%$ & 0.4 & 3247.8 & $27.6 \%$ & 7640.4 & 3205.4 & $26.0 \%$ & 10436.0 \\
\hline (H) & 18750.6 & $1.9 \%$ & 0.1 & 19340.1 & $5.1 \%$ & 9079.2 & 18924.9 & $2.9 \%$ & 13695.9 \\
\hline$(\mathrm{N})$ & 24212.8 & $7.2 \%$ & 5.0 & 24252.9 & $7.4 \%$ & 15405.3 & 23809.4 & $5.4 \%$ & 19713.0 \\
\hline$(\mathrm{S})$ & 443.4 & $2.6 \%$ & 0.1 & 465.3 & $7.7 \%$ & 47.472 & 455.5 & $5.4 \%$ & 79.9 \\
\hline & & $4.7 \%$ & 1.2 & & $11.1 \%$ & 11180.9 & & $8.8 \%$ & 15015.1 \\
\hline & & $4.4 \%$ & 1.0 & & $10.5 \%$ & 9325.3 & & $8.3 \%$ & 12525.9 \\
\hline
\end{tabular}

\begin{tabular}{||r||r|r|r|r|r|r||}
\hline \multicolumn{1}{||c||}{$(\mathrm{P})$} & \multicolumn{3}{|c|}{ H4 ${ }^{\text {dem }}$} & \multicolumn{3}{c|}{$\mathrm{H} 4^{\mathrm{H} 1}$} \\
& cost & gap & time $(\mathrm{s})$ & cost & gap & time (s) \\
\hline (I) & 9785.6 & $13.2 \%$ & 1034.6 & 8818.2 & $2.0 \%$ & 594.1 \\
(E) & 29121.6 & $9.8 \%$ & 14052.7 & 27495.2 & $3.6 \%$ & 11042.8 \\
(U) & 2880.3 & $13.2 \%$ & 1998.5 & 2649.1 & $4.1 \%$ & 1684.0 \\
(H) & 18968.8 & $3.1 \%$ & 3657.6 & 18646.4 & $1.4 \%$ & 1751.7 \\
(N) & 24151.9 & $6.9 \%$ & 3439.7 & 23383.9 & $3.5 \%$ & 1914.2 \\
\hline$(\mathrm{S})$ & 442.6 & $2.4 \%$ & 1.1 & 439.5 & $1.7 \%$ & 1 \\
\hline & & $9.2 \%$ & 4836.6 & & $2.9 \%$ & 3397.4 \\
& & $8.1 \%$ & 4030.7 & & $2.7 \%$ & 2831.3 \\
\hline
\end{tabular}

Table 3: Results from heuristics (H1, H2, H3, H4) and the literature (DP, DT)

\begin{tabular}{|c|c|c|c|c|c|c|c|}
\hline & DP & DT & H 1 & H 2 & H 3 & $\mathrm{H} 4^{\text {pdem }}$ & $\mathrm{H} 4^{\mathrm{H} 1}$ \\
\hline cost & 10130.6 & 11103.8 & 8840.9 & 9250.5 & 9009.6 & 9785.6 & 8818.2 \\
\hline HY & 174.0 & 787.4 & 0.0 & 253.9 & 227.0 & 28.43 & 164.2 \\
\hline $\mathrm{HL}$ & $44.0 \%$ & $42.9 \%$ & $36.6 \%$ & $35.4 \%$ & $38.2 \%$ & $37.52 \%$ & $37.9 \%$ \\
\hline
\end{tabular}

Table 4: Comparison of the solutions obtained on the profile INRETS

\subsection{Results analysis}

\subsubsection{Performance of the algorithms}

In Table 3 and Figure 8 it is shown that all proposed heuristics produced better solutions than the previous algorithms from the literature. The fuel consumption has been reduced significantly (more than 30\% in average), especially considering that the literature rated the solutions issued from the DP-based algorithm as optimal. H2 and H3 in particular, implemented using the same framework than the DP, validate the importance of calling into question the assumptions made in the literature when applying the DP in energy management for hybrid electric vehicles, as it is done in Section 4. Note that the DT also provided good results, better than the DP. 
All of the heuristics proposed obtained better solutions than the DT and one did so in a shorter computing time. On average, the DT solution costs have been improved by $19.13 \%$ ( $14.11 \%$ on real-world instances). This confirms that the predefined starting points considered in this paper produce better results than the linear constrained approximations proposed in the literature.

The values of the gap to LB show that the new best known solutions are proven to be less than $5 \%$ far from the optimal solutions on the real-world instances. The gap is also low on the synthetized profile SYNTH (around $1.7 \%$ ).

Regarding the computational time, there was a significant improvement of the proposed approaches not based on dynamic programming, comparing to the DP, but this is often the case in the literature. The difference here, is that better solutions were obtained in a shorter computing time. Of course, these computational times could be further reduced if all algorithms were implemented in $\mathrm{C}$ for example, but the solution values would not be impacted, as well as the order of magnitude of the computational times.

Finally, although the final SOC must be at least equal to the initial SOC, the costs of the best solutions found for all instances are on average $60 \%$ lower than the cost of using the FC only (UB). This was expected and has been used in the literature to highlight the benefits of hybrid architectures for vehicle propulsion. On one instance (URBAN) however, the weaknesses and assumptions from the DP identified in Section 4 led to a solution slightly worse than using the FC alone.

\subsubsection{Characteristics of the solution}

In Table 4 the characteristics of the solutions found by the different algorithms on the instance INRETS are summarized. No correlation could be found between the hydrogen storage (HY) and the solution quality or between the hybridization level and the solution quality.

In Figure 9, the SOC variation in the SE for the solution given by each method is shown. It seems to suggest that there exists a negative correlation between the fluctuations in the SE and the quality of the solutions in terms of fuel consumption. This may be explained by the fact that more often the energy is exchanged, more energy losses can potentially appear, especially at the lower portions of the efficiency curves. The validity domain plotted corresponds to the

case where $E_{\mathrm{SE}_{\text {final }}}=E_{\mathrm{SE}_{\text {init }}}$, but the heuristics also authorize $E_{\mathrm{SE}_{\text {final }}}>E_{\mathrm{SE}_{\text {init }}}$. This is the reason why $\mathrm{H} 2$ and $\mathrm{H} 3$ are able to produce curves that are outside the validity domain towards the end of the time horizon. However the minimum and the maximum state of charge $\left(E_{\mathrm{SE}_{\min }}\right.$ and $\left.E_{\mathrm{SE}_{\max }}\right)$ are always respected.

\subsection{Focus on Heuristic 4: randomization and multistart}

In order to illustrate the efficiency of the deterministic versions of $\mathrm{H} 4$ ( $\mathrm{H} 4^{\text {pdem }}$ and $\mathrm{H} 4^{\mathrm{H} 1}$ ) a multistart variant is considered: several iterations are performed, and on each iteration a random starting point is generated from which the gradient-based local search algorithm is applied. Note that the multistart version can be assimilated to a GRASP metaheuristic (Greedy Randomized Adaptative Search)([Feo and Resende, 1995]), although the starting point of the heuristic is not required to correspond to a feasible solution of the considered problem. 


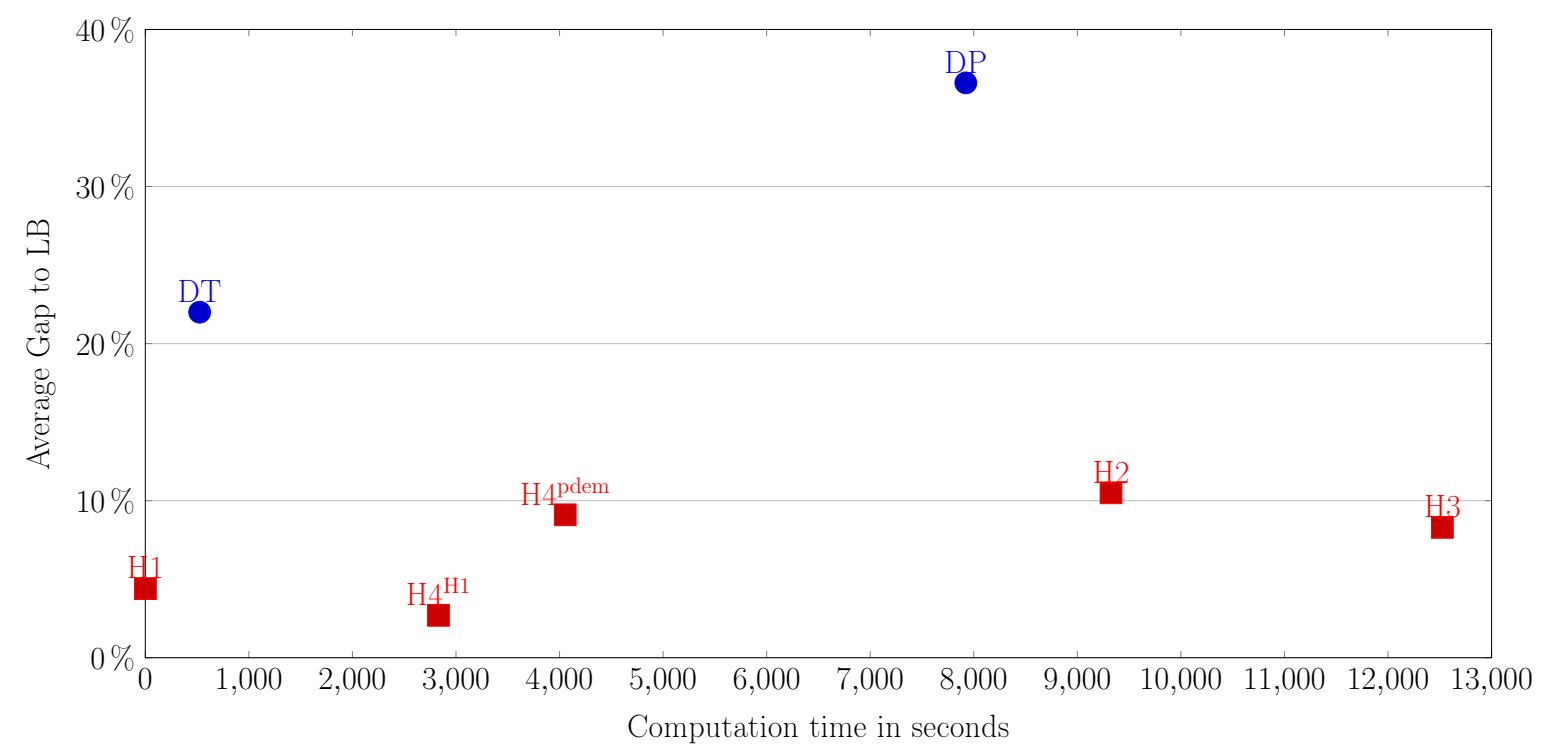

Figure 8: Comparison of the heuristics solutions: Average Gap to LB vs Computational time

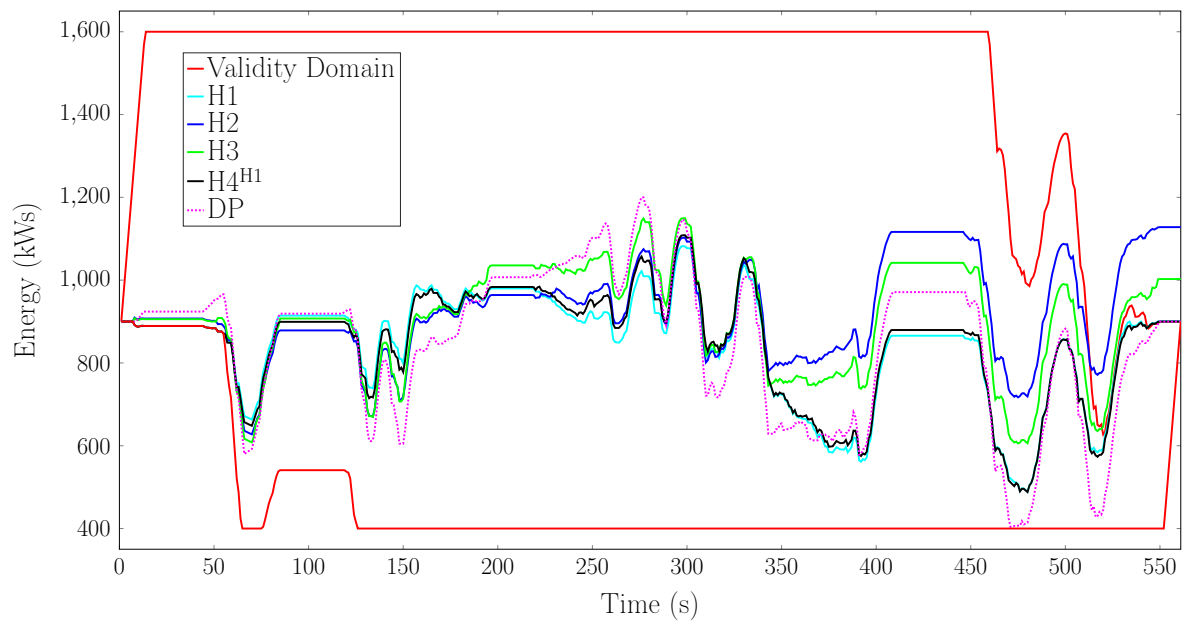

Figure 9: Energy variation in the storage element 


\subsubsection{Randomized multistart version}

A starting point of $\mathrm{H} 4$ is in fact a vector of dimension $2 n$ where $n$ is the number of considered instant-times. In $\mathrm{H}_{4}{ }^{\text {pdem }}$ for example, this vector is equal to the power demand at each instant-time, separated into positive and negative demands. For the randomized multistart version of H4, 200 random starting points were generated and the gradient-based optimization method was launched from each of these starting points to try to scan various parts of the solution space.

Let us focus on the mission profile INRETS for instance. The obtained results, illustrated on Figure 10(a), showed that most solution costs converged to a value around $9600 \mathrm{kWs}$ $\pm 200 \mathrm{kWs}$. However, with this strategy, it was possible after several iterations to find an interesting local minimum and the best solution obtained with this method had a cost of $8800 \mathrm{kWs}$. Nonetheless, using this strategy, SOC is under great fluctuations. As illustrated on Figure 9, this tends to be detrimental to the solution quality. To help avoiding such fluctuations, the random starting point generation was then modified as follows: generate each initial vector by picking random values between $-70 \mathrm{~kW}$ and $70 \mathrm{~kW}$, but before their introduction in the gradient-based solver, cancel (reset to 0) all values from it that are less than a predefined "decision value". The results of this strategy in function of the decision value are presented on Figure 10(b). It shows a significant improvement in the solutions costs when choosing a decision value just above 0 . In such case all negative values are set to 0 , which might be an explanation of the improvement. The final solution costs are consistently better than the purely randomized version for a decision value between 5 and 25. But despite this improvement, the multistart versions of $\mathrm{H} 4$ did not perform better than the deterministic versions $\mathrm{H} 4^{\text {pdem }}$ or $\mathrm{H} 4^{\mathrm{H} 1}$, and furthermore they increased the computational time.

\subsubsection{Perturbation of the deterministic starting point}

In Figure 11, it is shown that $\mathrm{H} 4^{\text {pdem }}$, taking a starting point equal to the power demand, uses the SE to absorb the very small and the very large power demand variations, allowing the FC to focus on providing energy in a threshold that corresponds to good FC efficiency. A small deviation from the starting point can lead to a large change in the energy profile but not necessarily in the fuel consumption. To confirm that, 35 starting points derived from the application of random variations on $P_{\text {dem }}$ have then been tested. In Figure 10(c), the cost of the obtained solutions from each of these 35 starting points on the instance INRETS are

plotted. No significant improvement was found in comparison to the result of $\mathrm{H} 4^{\text {pdem }}$.

\section{Conclusion}

Hybrid Electric Vehicles offer the possibility to recover the energy generated during the braking phases of any mission and allow to use it to minimize the fuel consumption and subsequently to minimize gas emissions. In this paper, four global optimization heuristics are developed for the distribution of powers between the fuel cell and the storage element. The objective is to minimize the cost of fuel consumption whilst satisfying the power demand.

The application of a gradient-based local search algorithm on two predefined starting points, provided the best results, outperforming state-of-the art methods from the literature 


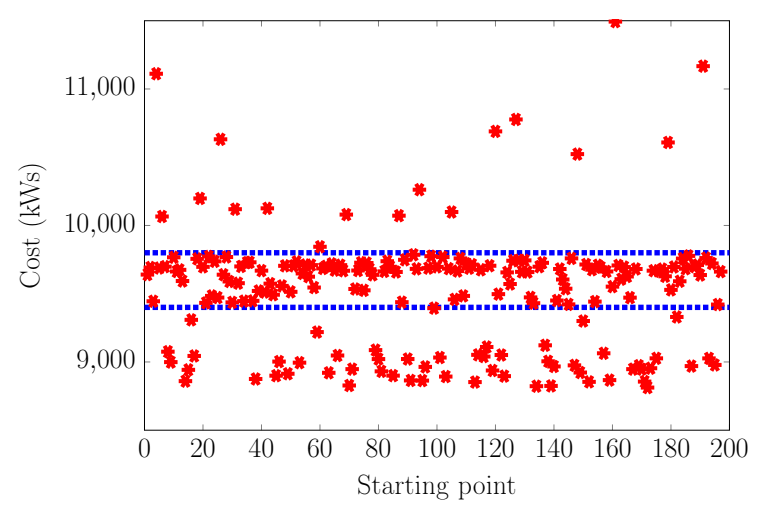

(a) Starting point $=$ random: 200 iterations

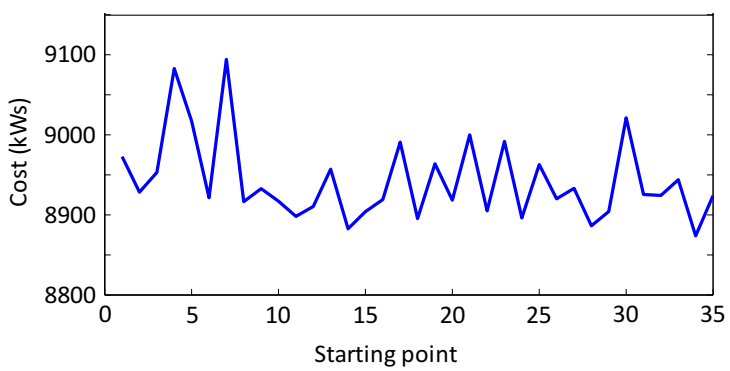

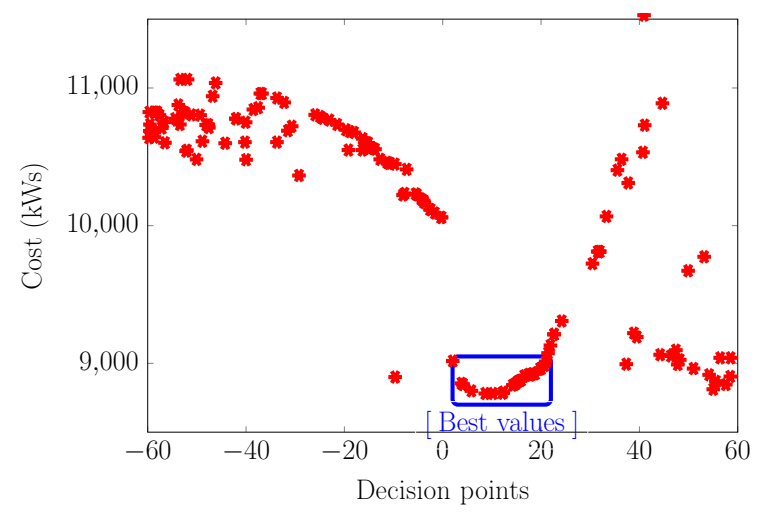

(b) Decision point vs solution cost

(c) Starting point $=$ random perturbation of $P_{\mathrm{dem}}: 35$ iterations

Figure 10: Results of multistart versions of H4 

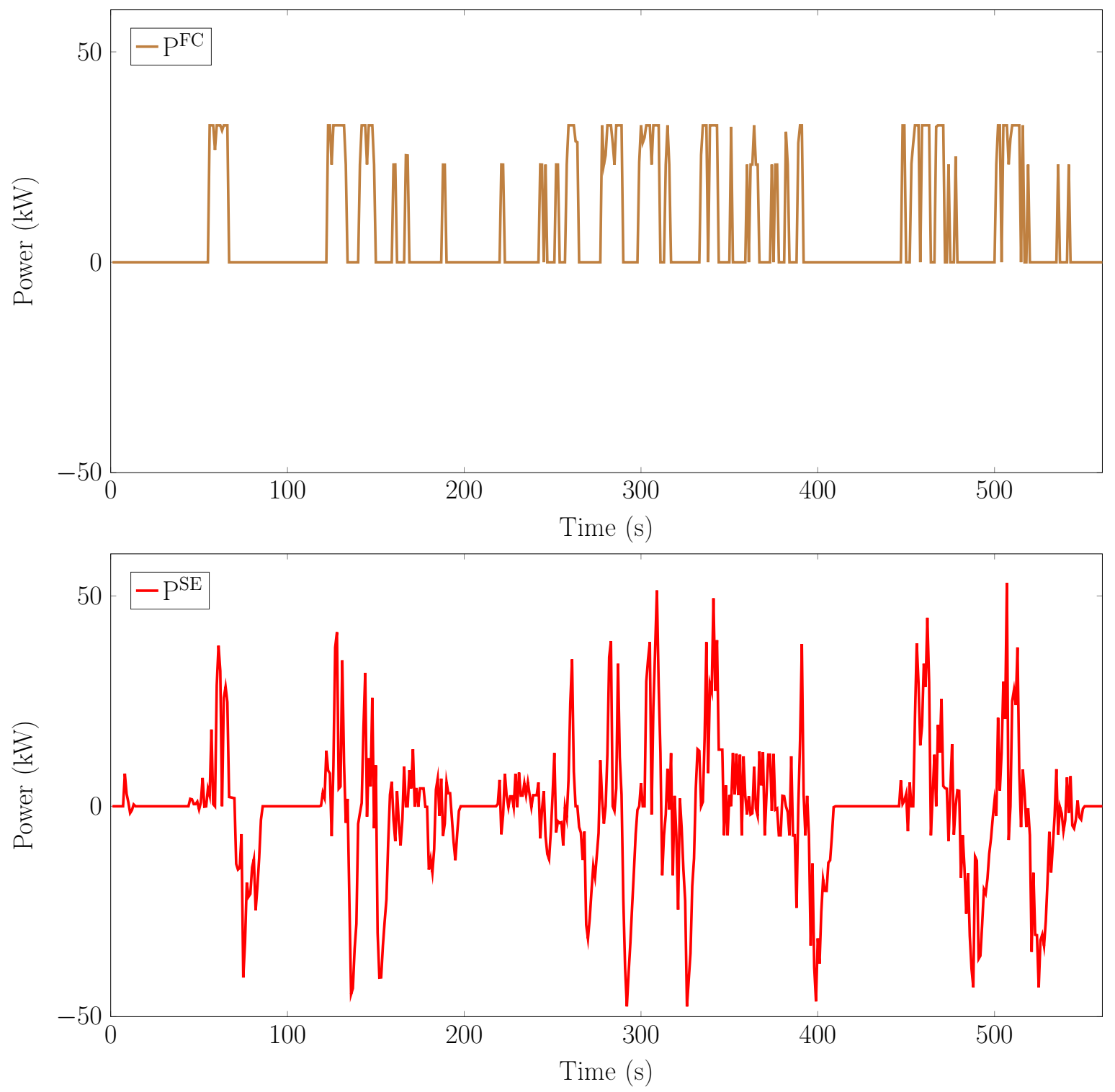

Figure 11: Solution obtained by $\mathrm{H} 4{ }^{\mathrm{H} 1}$ on INRETS: variations of $x_{i}\left(=P^{\mathrm{FC}}\right)$ and $y_{i}\left(=P^{\mathrm{SE}}\right)$ 
when no restricting assumption is made on any of the efficiency functions. Moreover, the newly computed lower bound helped proving that on the real-world power profiles available, the solutions obtained, if they were not optimal, were at most at $2 \%-4 \%$ far from the optimum. Moreover, if the computational time is crucial, one of the heuristics proposed can provide very efficient solutions (on average less than $5 \%$ far from the lower bound and thus from the optimal solution on the realistic profiles available) in a few seconds.

To summarize, the efficiency of the new approaches and resulting heuristics has been validated, both in terms of solutions quality (even in comparison to dynamic programming) and in computational time. Besides, the novelty in this work compared to previous researches is that the results are compared with lower bounds of consumption and not only to other upper bounds as it is usually done in the literature. For this reason, a guarantee can be given on the quality of the solutions for any mission profile for an hybrid-electric vehicle. Future work includes the use of robust or stochastic programming to handle power demand uncertainties if the power demand profile results from predictions or if it is subject to variations in an online context.

\section{Compliance with Ethical Standards}

- Conflict of Interest: The authors declare that they have no conflict of interest.

- Research involving Human Participants and/or Animals: not applicable to this publication

- Informed consent: not applicable to this publication

\section{References}

[Bernard et al., 2010] Bernard, J., Delprat, S., Guerra, T. M. and Büchi, F. N. (2010). Fuel efficient power management strategy for fuel cell hybrid powertrains. Control Engineering Practice 18, $408-417$.

[Bertsekas, 2011] Bertsekas, D. P. (2011). Dynamic Programming and Optimal Control vol. II, chapter 6 - Approximate Dynamic Programming, pp. 322-552. Nashua: ISBN 1-88652926-4, Massachusetts Institute of Technology, 3rd edition.

[Bolvashenkov et al., 2006] Bolvashenkov, I., Herzog, H.-G. and Engstle, A. (2006). Factor of hybridization as a design parameter for hybrid vehicles. In International Symposium on Power Electronics, Electrical Drives, Automation and Motion (SPEEDAM), pp. 926-929, IEEE.

[Bosman et al., 2012] Bosman, M. G. C., Bakker, V., Molderink, A., Hurink, J. L. and Smit, G. J. M. (2012). Planning the production of a fleet of domestic combined heat and power generators. European Journal of Operational Research 216, $140-151$.

[Brahma et al., 2000] Brahma, A., Guezennec, Y. and Rizzoni, G. (2000). Optimal energy management in series hybrid electric vehicles. In American Control Conference (ACC) vol. 1(6), pp. 60-64, IEEE. 
[Buecherl et al., 2009] Buecherl, D., Bolvashenkov, I. and Herzog, H. (2009). Verification of the optimum hybridization factor as design parameter of hybrid electric vehicles. In IEEE Vehicle Power and Propulsion Conference (VPPC) pp. 847-851, IEEE.

[Burke, 2007] Burke, A. F. (2007). Batteries and Ultracapacitors for Electric, Hybrid, and Fuel Cell Vehicles. Proceedings of the IEEE 95, 806-820.

[Chan, 2007] Chan, C. C. (2007). The State of the Art of Electric, Hybrid, and Fuel Cell Vehicles. Proceedings of the IEEE 95, 704-718.

[Feo and Resende, 1995] Feo, T. A. and Resende, M. G. C. (1995). Greedy Randomized Adaptive Search Procedures. Journal of Global Optimization 6, 109-133.

[Gao et al., 2009] Gao, J., Sun, F., He, H., Zhu, G. G. and Strangas, E. G. (2009). A Comparative Study of Supervisory Control Strategies for a Series Hybrid Electric Vehicle. In Asia-Pacific Power and Energy Engineering Conference (APPEEC) pp. 1-7, IEEE.

[Gaoua et al., 2013] Gaoua, Y., Caux, S. and Lopez, P. (2013). A Combinatorial Optimization Approach for the Electrical Energy Management in a Multi-Source System. In 2nd International Conference on Operations Research and Enterprise Systems (ICORES 2013) pp. 55-59, SciTePress.

[Hankache, 2008] Hankache, W. (2008). Gestion Optimisée de l'énergie électrique d'un Groupe Electrogène Hybride à Pile à Combustible. PhD thesis, Institut National Polytechnique de Toulouse.

[He et al., 2012] He, Y., Chowdhury, M., Pisu, P. and Ma, Y. (2012). An energy optimization strategy for power-split drivetrain plug-in hybrid electric vehicles. Transportation Research Part C: Emerging Technologies 22, $29-41$.

[Hofman et al., 2007] Hofman, T., Steinbuch, M., Van Druten, R. and Serrarens, A. (2007). Rule-based energy management strategies for hybrid vehicles. International Journal of Electric and Hybrid Vehicles 1, 71-94.

[Horst and Tuy, 2000] Horst, R. and Tuy, H. (2000). Global Optimization: Deterministic Approaches. 3rd edition, ISBN 9783540610380, Springer. 728 pages.

[Jacomino and Le, 2012] Jacomino, M. and Le, M. (2012). Robust energy planning in buildings with energy and comfort costs. A Quarterly Journal of Operations Research (4OR) 10, 81-103.

[Jalil et al., 1997] Jalil, N., Kheir, N. A. and Salman, M. (1997). A rule-based energy management strategy for a series hybrid vehicle. In American Control Conference (ACC) pp. 689 - 693, IEEE.

[Kim et al., 2011] Kim, N., Cha, S. and Peng, H. (2011). Optimal Control of Hybrid Electric Vehicles Based on Pontryagin's Minimum Principle. Control Systems Technology, IEEE Transactions on 19, 1279-1287. 
[Lukic and Emadi, 2004] Lukic, S. M. and Emadi, A. (2004). Effects of drivetrain hybridization on fuel economy and dynamic performance of parallel hybrid electric vehicles. Vehicular Technology, IEEE Transactions on 53, 385-389.

[Mathworks, 2013] Mathworks (accessed on March 27th, 2013). optimization toolbox website. http://www.mathworks.fr/help/toolbox/optim/ug/fmincon.html.

[Neffati et al., 2012] Neffati, A., Caux, S. and Fadel, M. (2012). Fuzzy switching of fuzzy rules for energy management in HEVs. In 8th Power Plant and Power System Control Symposium (PP\&PSC) pp. 663-668,.

[Ngo et al., 2010] Ngo, D. V., Hofman, T., Steinbuch, M. and Serrarens, A. F. A. (2010). An Optimal Control-Based Algorithm for Hybrid Electric Vehicle using Preview Route Information. In American Control Conference (ACC) pp. 5818-5823, IEEE.

[Paganelli et al., 2002] Paganelli, G., Delprat, S., Guerra, T.-M., Rimaux, J. and Santin, J. J. (2002). Equivalent consumption minimization strategy for parallel hybrid powertrains. In IEEE 55th Vehicular Technology Conference (VTC) vol. 4, pp. 2076-2081, IEEE.

[Pérez and García, 2010] Pérez, L. and García, G. O. (2010). State Constrained Optimal Control Applied to Supervisory Control in HEVs, Oil \& Gas Science and Technology. Revue de l'Institut Français du Pétrole 65, 191-201.

[Pérez et al., 2006] Pérez, L. V., Bossio, G. R., Moitre, D. and García, G. O. (2006). Optimization of power management in an hybrid electric vehicle using dynamic programming. Mathematics and Computers in Simulation 73, 244-254.

[Pérez and Pilotta, 2009] Pérez, L. V. and Pilotta, E. A. (2009). Optimal power split in a hybrid electric vehicle using direct transcription of an optimal control problem. Mathematics and Computers in Simulation 79, 1959-1970.

[Pisu and Rizzoni, 2007] Pisu, P. and Rizzoni, G. (2007). A Comparative Study Of Supervisory Control Strategies for Hybrid Electric Vehicles. Control Systems Technology, IEEE Transactions on $15,506-518$.

[Shen et al., 2011] Shen, C., Shan, P. and Gao, T. (2011). A Comprehensive Overview of Hybrid Electric Vehicles. International Journal of Vehicular Technology 2011, 7 pages.

[Vis, 2006] Vis, I. F. A. (2006). Survey of research in the design and control of automated guided vehicle systems. European Journal of Operational Research 170, 677-709.

[Yu et al., 2009] Yu, Y.-B., Wang, Q.-N., Min, H.-T. and Wang, P.-Y.and Hao, C.-G. (2009). Control strategy optimization using dynamic programming method for synergic electric system on hybrid electric vehicle. Natural Science 1, 222-228.

[Zadeh, 1965] Zadeh, L. (1965). Fuzzy sets. Information and Control 8, 338-353. 